\title{
NUTRIENTES NA FITOMASSA DE CAPIM-MARANDU EM FUNÇÃO DE FONTES E DOSES DE NITROGÊNIO ${ }^{1}$
}

\author{
Nutrient content in marandu grass biomass due to nitrogen sources and levels
}

\author{
Ana Cândida Primavesi², Odo Primavesi ${ }^{2}$, Luciano de Almeida Corrêa ${ }^{2}$ \\ Aliomar Gabriel da Silva ${ }^{2}$, Heitor Cantarella ${ }^{3}$
}

\begin{abstract}
RESUMO
Em pastagens manejadas intensivamente, que recebem altas doses de nutrientes, é necessário conhecer sua extração para auxiliar as recomendações de adubação. Este trabalho teve a finalidade de avaliar os nutrientes extraídos pela fitomassa de capimmarandu (Brachiaria brizantha (Hochst ex. A. Rich.) Stapf cv. Marandu) submetido a fontes e doses de $\mathrm{N}$ e seu efeito na recuperação aparente do N, em experimento conduzido em Latossolo Vermelho Distrófico típico, sob condições tropicais. Foram aplicadas três doses de N: 50, 100 e $200 \mathrm{~kg} \mathrm{ha}^{-1}$ corte $^{-1}$, nas formas de uréia e de nitrato de amônio (NA), após quatro cortes consecutivos na estação chuvosa, além da testemunha. Houve aumento na extração dos nutrientes com doses crescentes de $\mathrm{N}$, com valores elevados para $\mathrm{K}$ e $\mathrm{N}$. As extrações dos macronutrientes foram maiores para $\mathrm{K}$ seguidas de $\mathrm{N}, \mathrm{Ca}, \mathrm{Mg}, \mathrm{P}$ e $\mathrm{S}$ e dos micronutrientes na seguinte ordem decrescente: Fe, Mn, Zn e Cu. A recuperação média do N, pela forragem, de todas as doses da uréia foi de $84 \%$ da obtida com o NA, para o qual variou entre 38 e $51 \%$. Em capim-marandu com produções elevadas de forragem, a extração de K é grande e a recuperação média de $\mathrm{N}$ da uréia é inferior à média do nitrato de amônio.
\end{abstract}

Termos para indexação: Brachiaria brizantha, extração de nutrientes, nitrato de amônio, teores de nutrientes, uréia.

\begin{abstract}
Data about nutrient extraction in pastures receiving high rates of nutrients are important to help to define fertilizer recommendations. The present work had the objective of evaluating nutrient extraction and $\mathrm{N}$ recovery by marandu grass (Brachiaria brizantha (Hochst ex. A. Rich.) Stapf cv. Marandu) treated with different sources and rates of N, grown on a dark red latosol (Hapludox), under tropical conditions, in Brazil. Three rates of $\mathrm{N}$ were surface-applied: 50, 100 and $200 \mathrm{~kg} \mathrm{ha}^{-1}$ cutting ${ }^{-1} \mathrm{as} \mathrm{urea} \mathrm{or}^{-}$ ammonium nitrate, after four consecutive cuttings, during the rainy season. Nutrient extraction increased with increasing nitrogen rates, with high values mainly for $\mathrm{K}$ and $\mathrm{N}$. When forage yield was high (treatment with $400 \mathrm{~kg} \mathrm{ha}^{-1} \mathrm{year}^{-1}$ of $\mathrm{N}$ ) and for both fertilizer, macronutrient extraction was greater for $\mathrm{K}$ and $\mathrm{N}$, followed by $\mathrm{Ca}, \mathrm{Mg}, \mathrm{P}$, and $\mathrm{S}$. Micronutrient extraction decreased in the following order: $\mathrm{Fe}, \mathrm{Mn}, \mathrm{Zn}$, and $\mathrm{Cu}$. Mean $\mathrm{N}$ recovery, by forage, from urea was of about $84 \%$ of that of ammonium nitrate, which varied from 38 to $51 \%$ of applied N. Large amounts of K are extract for marandu grass with high forage yield, and mean urea-N recovery is lower than for ammonium nitrate-N.
\end{abstract}

Index terms: Ammonium nitrate, Brachiaria brizantha, nutrient Content, nutrient extraction, urea.

(Recebido para publicação em 20 de setembro de 2005 e aprovado em 16 de fevereiro de 2006)

\section{INTRODUÇÃO}

As pastagens de gramíneas tropicais corretamente estabelecidas e adequadamente manejadas e adubadas constituem fonte de alimento para bovinos que pode ser produzido economicamente e em grande quantidade. Sistemas intensivos de produção de bovinos demandam tecnologias e insumos para torná-los mais eficientes, competitivos e lucrativos .

Fertilizantes e corretivos, corretamente aplicados, são fatores determinantes no aumento da produtividade das forrageiras com potencial de resposta à aplicação de adubos, principalmente do nitrogênio $(\mathrm{N})$.

$\mathrm{O}$ uso eficiente da pastagem, em sistemas intensivos de produção, é dependente de concentrações adequadas de elementos minerais na forragem (HOPKINS et al., 1994). Entretanto, as conseqüências da intensificação do manejo de pastagens na composição mineral da forragem são bem menos compreendidas, em particular o efeito relativo do aumento da aplicação de fertilizantes nitrogenados na concentração de vários nutrientes.

De todos os nutrientes, o $\mathrm{N}$ é quantitativamente o mais importante para o crescimento das plantas (ENGELS \& MARSCHNER, 1995) e de pastagens estabelecidas com gramíneas (MONTEIRO et al., 2004), e o segundo fator mais limitante (o primeiro sendo a água) para o desenvolvimento das forrageiras (JARVIS et al., 1995).

Para um bom manejo da adubação, principalmente em sistema intensivo de produção, torna-se importante conhecer a necessidade de nutrientes das plantas

1 Trabalho financiado pelo Convênio Embrapa/Petrobrás.

${ }^{2}$ Embrapa Pecuária Sudeste - Cx. P. 339 - 13560-970 - São Carlos, SP - anacprima@yahoo.com.br, odo@cppse.embrapa.br luciano@cppse.embrapa.br, aliomar.silva@embrapa.br

${ }^{3}$ Instituto Agronômico de Campinas, Centro de Solos e Recursos Agroambientais - Cx. P. 28 - 13001-970 - Campinas, SP - cantarella@iac.sp.gov.br 
forrageiras e, conseqüentemente, sua capacidade de extraílos do solo (LUZ et al., 2001).

Entretanto, aplicações elevadas de $\mathrm{N}$ podem resultar em elevação do teor protéico, redução nos teores de matéria seca (MS) (WHITEHEAD, 1995), fósforo (P) e potássio (K) e outras interações entre nutrientes minerais no solo e planta. $\mathrm{O}$ efeito dos fertilizantes nitrogenados no pH do solo é particularmente importante na absorção do ferro $(\mathrm{Fe})$, manganês $(\mathrm{Mn})$ e cobalto $(\mathrm{Co})$ e, portanto, influencia a composição mineral da forrageira (WHITEHEAD, 1995).

Os resultados de pesquisa em relação às perdas de $\mathrm{N}$ são muito variados, principalmente porque o $\mathrm{N}$ proveniente da uréia é muito susceptível às perdas por volatilização e, conseqüentemente, mais sensível às condições do manejo de aplicação. Em pastagens manejadas intensivamente, onde se usam doses elevadas de $\mathrm{N}$, conhecer a recuperação do $\mathrm{N}$ do fertilizante pelas plantas torna-se importante para maximizar a eficiência do seu uso e minimizar o impacto ambiental. A recuperação aparente do $\mathrm{N}$ do fertilizante, isto é, a diferença do $\mathrm{N}$ absorvido por plantas de parcelas adubadas em relação às plantas de parcelas não adubadas, apresenta a conveniência de ser de fácil estimativa e de baixo custo, pois utiliza apenas o teor de $\mathrm{N}$ total da planta e a massa seca da forragem.

Realizou-se o presente trabalho com a finalidade de avaliar os nutrientes extraídos pela fitomassa de capimmarandu [Brachiaria brizantha (Hochst ex. A. Rich.) Stapf cv. Marandu], submetido a fontes e doses de N, e o efeito dessas na recuperação aparente do $\mathrm{N}$ de dois adubos nitrogenados, em experimento conduzido em Latossolo Vermelho Distrófico típico (LVd), para auxiliar as recomendações de adubação de pastagens exploradas intensivamente.

\section{MATERIAL E MÉTODOS}

O experimento foi conduzido de 20 de novembro de 2000 a maio de 2001, em pastagem de capim-marandu, em Latossolo Vermelho Distrófico típico, na fazenda Canchim, região de São Carlos, SP, Brasil (22 $2^{\circ} 1^{\prime}$ S e $47^{\circ} 54^{\prime} \mathrm{W}$, altitude de $836 \mathrm{~m}$ ), sob clima tropical de altitude.

As características químicas do solo, na camada de 0-20 cm, no início do experimento, foram: $\mathrm{pH} \mathrm{em} \mathrm{CaCl}_{2}=$ 5,5; M.O. $=55 \mathrm{~g} \mathrm{dm}^{-3} ;$ P-resina $=19 \mathrm{mg} \mathrm{dm}^{-3} ; \mathrm{K}=7,0 \mathrm{mmol}_{\mathrm{c}}$ $\mathrm{dm}^{-3} ; \mathrm{Ca}=54 \mathrm{mmol}_{\mathrm{c}} \mathrm{dm}^{-3} ; \mathrm{Mg}=21 \mathrm{mmol}_{\mathrm{c}} \mathrm{dm}^{-3} ; \mathrm{CTC}=116$ $\mathrm{mmol}_{\mathrm{c}} \mathrm{dm}^{-3} ; \mathrm{V}=70 \%$, e as características físicas do solo: areia $=559 \mathrm{~g} \mathrm{~kg}^{-1}$; argila $=400 \mathrm{~g} \mathrm{~kg}^{-1}$; silte $=40 \mathrm{~g} \mathrm{~kg}^{-1}$.
$\mathrm{Na}$ instalação do experimento foram aplicados 50 $\mathrm{kg} \mathrm{ha}^{-1}$ de $\mathrm{P}_{2} \mathrm{O}_{5}$, como superfosfato simples, e $30 \mathrm{~kg} \mathrm{ha}^{-1} \mathrm{de}$ micronutrientes FTE BR-12. O potássio, na forma de $\mathrm{KCl}$, foi aplicado por ocasião das adubações nitrogenadas, nas quantidades totais de $240 \mathrm{~kg} \mathrm{ha}^{-1} \mathrm{de} \mathrm{K}_{2} \mathrm{O}$, nos tratamentos testemunha e com $200 \mathrm{~kg} \mathrm{ha}^{-1}$ ano $^{-1}$ de N, e de $420 \mathrm{~kg} \mathrm{ha}^{-1}$ de $\mathrm{K}_{2} \mathrm{O}$, nos tratamentos com 400 e $800 \mathrm{~kg} \mathrm{ha}^{-1}$ ano ${ }^{-1}$ de N.

$\mathrm{O}$ delineamento experimental foi o de blocos casualizados com sete tratamentos organizados em esquema fatorial $(2 \times 3)+1$ (duas fontes de $\mathrm{N}$ : uréia e nitrato de amônio (NA) e três doses de N: 50,100 e $200 \mathrm{~kg}$ ha $^{-1}$ corte $^{-1}$ ), uma testemunha sem adubo nitrogenado, com quatro repetições (GATES, 1991). O N foi aplicado em quatro períodos (após o corte de uniformização e após os demais cortes, até o terceiro e penúltimo corte) durante a época das águas.

As parcelas apresentavam área de $16 \mathrm{~m}^{2}(4 \mathrm{x} 4 \mathrm{~m})$, sendo utilizada uma área útil de $6 \mathrm{~m}^{2}$, para avaliação da produção de forragem. Os cortes foram feitos a intervalos de 43 dias, a $10 \mathrm{~cm}$ da superfície do solo. Após a pesagem da matéria verde foi separada uma amostra com $500 \mathrm{~g}$, colocada em estufa de circulação forçada de ar, à temperatura de $60^{\circ} \mathrm{C}$, até peso constante, para determinação do teor de água e posterior cálculo do peso da MS. Foi determinado o teor de minerais na MS da forragem (MALAVOLTA et al., 1997) e calculada a extração de cada elemento.

A extração dos nutrientes foi calculada pela fórmula; Nutriente (ext) $\left(\mathrm{kg} \mathrm{ha}^{-1}\right)=0,001 \mathrm{x}$ [matéria seca $\left(\mathrm{kg} \mathrm{ha}^{-1}\right) \mathrm{x}$ teor do nutriente $\left(\mathrm{g} \mathrm{kg}^{-1}\right)$ ].

As chuvas ocorridas nos quatro períodos consecutivos de produção de forragem foram respectivamente: 199,0;149,1; 134,3 e 43,8 mm.

A recuperação aparente do $\mathrm{N}(\mathrm{Nrec}), \mathrm{em} \%$, foi calculada pela fórmula: $\mathrm{Nrec}=100 \times$ [(Nextr na parcela fertilizada - Nextr na parcela testemunha)/dose de $\mathrm{N}$ aplicada]. A extração de $\mathrm{N}$ (Nextr) foi calculada pela fórmula: Nextr $=0,001$ MS x TN; em que Nextr $=\mathrm{N}$ extraído em $\mathrm{kg}$ ha 1; MS= matéria seca em kg ha' ${ }^{-1} \mathrm{TN}=$ teor de $\mathrm{N} \mathrm{em} \mathrm{g} \mathrm{kg}^{-1}$. A quantidade de $\mathrm{N}$ na forragem das parcelas não adubadas foi utilizada para estimar o suprimento de $\mathrm{N}$ proveniente do solo e da atmosfera.

Foi realizada a análise de variância, com desdobramento e avaliação do peso das componentes do primeiro ao quarto grau, e usando-se o teste $\mathrm{F}$ para comparar as médias das fontes de $\mathrm{N}$, bem como ajustadas equações de regressão linear simples e quadrática para as curvas de teores e de extração de nutrientes em função das doses de $\mathrm{N}$ aplicadas. 


\section{RESULTADOS E DISCUSSÃO}

A análise de variância mostrou diferenças significativas $(\mathrm{P}<0,05)$ para o efeito de fontes de $\mathrm{N}$ sobre a concentração de todos os nutrientes, exceto $\mathrm{P}, \mathrm{K}, \mathrm{Mn}$ e $\mathrm{Fe}$, na parte aérea do capim-marandu. Ocorreu interação fontes $\mathrm{x}$ doses para $\mathrm{N}, \mathrm{P}, \mathrm{K}, \mathrm{Ca}, \mathrm{Mg}, \mathrm{Cu}, \mathrm{Zn}$ e $\mathrm{Mn}$, expressa por diferenças nas curvas de resposta (Tabela 1 ).

Os teores de $\mathrm{N}$ na planta aumentaram com as doses de $\mathrm{N}$, das duas fontes, de forma linear com a uréia e quadrática com o NA (Tabela 1). Com a uréia, os teores de $\mathrm{N}\left(\mathrm{g} \mathrm{kg}^{-1}\right)$ nas plantas variaram de 17 a 24 , e com o NA de 18 a 26 , sendo a faixa adequada de 13 a 20 (WERNER et al., 1996). No tratamento $200 \mathrm{~kg} \mathrm{ha}^{-1}$ corte $^{-1} \mathrm{de} \mathrm{N}$, o teor de $\mathrm{N}$ se mostrou maior que o adequado, confirmado pelo acúmulo de nitrato na forragem. O nitrato $\left(\mathrm{NO}_{3}^{-}\right)$é a forma inorgânica de $\mathrm{N}$ que se acumula na planta quando o suprimento excede o requerimento para o crescimento.
As diferenças entre fontes podem ser explicadas pelas perdas de $\mathrm{N}-\mathrm{NH}_{3}$ da uréia. Ocorreu aumento nos teores

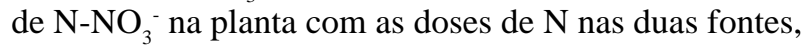
sendo maior com o NA. Verifica-se no tratamento $200 \mathrm{~kg}$ ha $^{-1}$ corte $^{-1}$ de $\mathrm{N}$ em que a resposta em produção foi mais reduzida, que o teor de $\mathrm{N}_{-} \mathrm{NO}_{3}$ - foi maior, indicando que não compensa aumentar as doses de $\mathrm{N}$, pois embora $\mathrm{o}$ teor de $\mathrm{N}$ na planta ainda esteja aumentando, o $\mathrm{N}_{-} \mathrm{NO}_{3}{ }_{3}^{-}$já está acumulando, embora ainda na faixa permissível para alimentação animal - 3.400 a $4.500 \mathrm{mg} \mathrm{kg}^{-1}$ (WHITEHEAD, 1995). Esse fato também permite explicar porque não se observou o efeito de diluição para teores de $\mathrm{N}$, que continuaram aumentando com a maior produção de MS. Tem-se observado que o K aumenta a eficiência de uso do $\mathrm{N}$. Com teores baixos de $\mathrm{K}$ nas plantas, os compostos de N solúvel acumulam-se (COUTINHO et al., 2004). Tal fato não foi observado neste experimento.

TABELA 1 - Teores de nutrientes e relação N:S na forragem de capim-marandu em função de fontes e doses de $\mathrm{N}^{(1)}$.

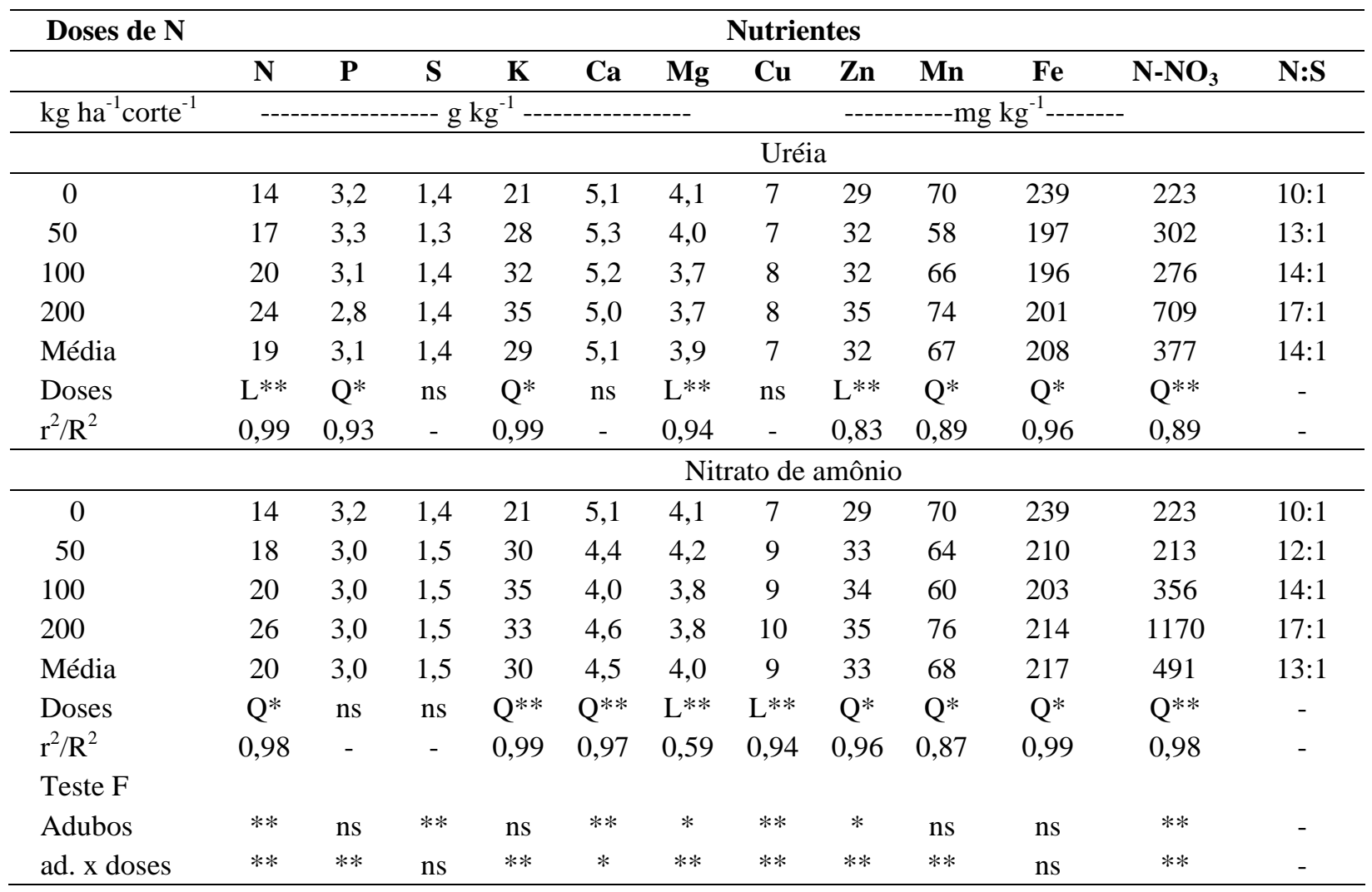

${ }^{(1)}=$ média de quatro cortes. ${ }^{\text {ns }}=$ não-significativo. $*$ ou $* *=$ significância do teste $\mathrm{F}$ no nível de $5 \%$ e $1 \%$. Para doses, apresentada a curva de melhor ajuste $\left(\mathrm{L}=\right.$ Linear simples ou $\mathrm{Q}=$ Quadrática) e o valor de $\mathrm{r}^{2}$ (linear) e $\mathrm{R}^{2}$ (quadrático).

Ciênc. agrotec., Lavras, v. 30, n. 3, p. 562-568, maio./jun., 2006 
Com o S é necessário considerar o seu teor e a relação N:S no tecido vegetal (MONTEIRO et al., 2004). Batista (2002) verificou em capim-marandu que a produção de MS dependeu do equilíbrio no fornecimento desses dois nutrientes. Os teores de $\mathrm{S}$ não variaram com as doses de $\mathrm{N}$ em ambas as fontes, mas ocorreu variação entre as fontes, com teores superiores para o NA, mas com valores dentro da faixa dos teores adequados - 0,8 a $2,5 \mathrm{~g} \mathrm{~kg}^{-1}$ (WERNER et al., 1996). Faquin et al. (2000) obtiveram com capim-marandu, teores entre 0,4 e $0,5 \mathrm{~g} \mathrm{~kg}^{-1} \mathrm{de} \mathrm{S}$, no tratamento que não recebeu Jones \& Watson (1991) relataram para as gramíneas relações N:S de 10:1; Scott et al. (1983) informam que, em geral, valores de N:S acima de 14:1 indicam deficiência de $S$. No presente experimento a relação N:S variou de 10:1 a 17:1, tendo sido adicionado ao solo $30 \mathrm{~kg} \mathrm{ha}^{-1}$ de $\mathrm{S}$. A relação $\mathrm{N}: \mathrm{S}$ aumentou com o acréscimo das doses de $\mathrm{N}$, o que era esperado porque a dose de $\mathrm{S}$ foi fixa, o que refletiu nos teores de $\mathrm{S}$ de todos os tratamentos, inclusive no tratamento sem N. Nos tratamentos sem N e $200 \mathrm{~kg} \mathrm{ha}^{-1}$ corte $^{-1}$ de N, a relação N:S foi, respectivamente, 10:1 e 17:1, indicando que a quantidade aplicada de $30 \mathrm{~kg} \mathrm{ha}^{-1}$ de $\mathrm{S}$ foi suficiente para manter o equilíbrio entre $\mathrm{N}$ e $\mathrm{S}$. Isso confirma que, para pastagens exploradas intensivamente e onde se aplica $\mathrm{N}$ em grandes quantidades, as doses 30 a $90 \mathrm{~kg} \mathrm{ha}^{-1} \mathrm{de} \mathrm{S}$, recomendadas por ano, são suficientes, devendo ser parceladas sempre que se usar mais que $30 \mathrm{~kg} \mathrm{ha}^{-1}$ de $\mathrm{S}$ (MONTEIRO et al., 2004).

Os teores de K na MS das forrageiras variam com a disponibilidade do nutriente no solo. Em capim-marandu esse teor tem variado entre 4,3 e 28,0 $\mathrm{g} \mathrm{kg}^{-1}$ (MONTEIRO et al., 1995). Os teores de $\mathrm{K}\left(\mathrm{g} \mathrm{kg}^{-1}\right)$ aumentaram com as doses de $\mathrm{N}$ e variaram de 28 a 35, e nos tratamentos 100 e $200 \mathrm{~kg}$ ha $^{-1}$ corte $^{-1}$ de $\mathrm{N}$ ficaram acima da faixa adequada para essa forrageira - 12 a 30 (WERNER et al., 1996) - e 22 a 29 nas folhas novas (MATTOS, 1997), e muito acima do exigido por vacas leiteiras com $400 \mathrm{~kg}$ de peso vivo e produções de 7 a $20 \mathrm{~L} \mathrm{dia}^{-1}$, que é de $9 \mathrm{~g} \mathrm{~kg}^{-1}$ de K (NRC, 2001). Com o aumento das produções de MS, nos tratamentos 100 e 200 $\mathrm{kg} \mathrm{ha}^{-1}$ corte $^{-1}$ de $\mathrm{N}$, com a uréia não ocorreu diminuição no teor de $\mathrm{K}$, possivelmente porque maior quantidade de $\mathrm{K}$ foi usada e a gramínea cresceu menos que nos mesmos tratamentos com NA, nos quais o teor de $\mathrm{K}$ decresceu no tratamento $200 \mathrm{~kg} \mathrm{ha}^{-1}$ corte $^{-1}$ de $\mathrm{N}$, provavelmente pelo efeito de diluição.

Os teores de $\mathrm{Ca}$ e $\mathrm{Mg}$ da forragem ficaram dentro da faixa adequada - Ca - 3,0 a 6,0 $\mathrm{g} \mathrm{kg}^{-1} \mathrm{e} \mathrm{Mg}-1,5$ a $4,0 \mathrm{~g} \mathrm{~kg}^{-1}$ (WERNER et al., 1996). Com o aumento das doses de N, os teores de Ca sofreram ligeira redução quando se utilizou uréia e aumentaram discretamente com NA, e os de $\mathrm{Mg}$ diminuíram com os dois adubos nas doses maiores de $\mathrm{N}$, onde foi aplicado mais $\mathrm{K}$. A adição de $\mathrm{K}$ aumenta seus teores na planta e diminui os de $\mathrm{Ca}$ ou $\mathrm{Mg}$ em quantidades equivalentes (MATTOS et al., 2002). Normalmente o Mg não é usado em adubações, mas na calagem, e o uso de $\mathrm{K}$ em doses elevadas pode levar à deficiência de $\mathrm{Mg}$ (MARSCHNER, 1995). O efeito dos adubos nitrogenados no teor de $\mathrm{Mg}$ pode ter sido influenciado pela maior quantidade de $\mathrm{K}$ usada nos tratamentos 100 e $200 \mathrm{~kg} \mathrm{ha}^{-1}$ corte $^{-1}$ de N, uma vez que, em princípio, a maior absorção de $\mathrm{K}$ conduz à redução do teor de $\mathrm{Mg}$. Como, geralmente, o efeito do adubo nitrogenado sobre a produção de MS é maior que o dos adubos potássicos (HOPKINS et al., 1994), embora o teor de $\mathrm{Mg}$ tenha diminuído com o aumento das doses de $\mathrm{N}$, o teor de $\mathrm{Mg}$ e a relação $\mathrm{K} / \mathrm{Mg}$ se mantiveram na faixa de 7 a 10, considerada adequada por Malavolta (1980).

O N estimula a absorção e translocação do $\mathrm{P}$ (ANDREW \& ROBINS, 1971). Com o NA os teores de $\mathrm{P}$ permaneceram adequados e com a uréia ficaram acima - 0,8 a 3,0 $\mathrm{g} \mathrm{kg}^{-1}$ (WERNER et al., 1996). Os teores de P não aumentaram com doses de $\mathrm{N}$ na forma de NA e diminuíram com as doses de $\mathrm{N}$ na forma de uréia.

Os teores de $\mathrm{Cu}$ aumentaram com as doses de $\mathrm{N}$, com NA, o que também foi verificado por Andrade et al. (1996). Com os teores de Zn, a resposta foi linear com uréia e quadrática com NA. Com Mn e Fe, nas duas fontes de N, a resposta foi quadrática. Os teores $\left(\mathrm{mg} \mathrm{kg}^{-1}\right)$ de $\mathrm{Cu}(7 \mathrm{a}$ 10), Zn (29 a 35), Mn (58 a 76) e Fe (196 a 239) ficaram na faixa adequada (WERNER et al., 1996).

A extração dos nutrientes foi linear para N, Mn e $\mathrm{Fe}$, quadrática para $\mathrm{P}, \mathrm{Ca}$ e $\mathrm{Mg}$ para ambas fontes. Para S, $\mathrm{K}, \mathrm{Cu}$ e $\mathrm{Zn}$ a extração foi linear com uréia e quadrática com NA. Ocorreu interação para a extração de todos os nutrientes (Tabela 2). Comparando a extração de nutrientes na dose $400 \mathrm{~kg} \mathrm{ha}^{-1}$ ano $^{-1}$ de $\mathrm{N}$, em relação à testemunha, verificou-se aumento, respectivamente, para uréia e NA, de 6,7 e 8,5 vezes para $\mathrm{N} ; 4,8$ e 6,0 para $\mathrm{P} ; 4,3$ a 5,7 para $\mathrm{S}$; 7,5 e 9,3 para K; 4,7 e 4,9 para Ca; 4,3 e 5,4 para Mg; 5,0 e 6,8 para $\mathrm{Cu} ; 5,3$ e 6,9 para Zn; 4,4 e 5,0 para Mn e 4,9 e 3,8 para Fe. A extração dos nutrientes foi grande e cresceu com o aumento da produção de forragem. Houve extração bem maior de $\mathrm{K}$ em relação ao $\mathrm{N}$, mesmo no tratamento sem $\mathrm{N}$ mas que recebeu $\mathrm{K}$, indicando que o capim-marandu extrai muito K do solo. Primavesi et al. (2004) verificaram com capim-coastcross extrações semelhantes de K e de N. Nos tratamentos 400 e $800 \mathrm{~kg} \mathrm{ha}^{-1}$ ano $^{-1}$ de N, a maior extração de $\mathrm{K}$ refletiu a maior quantidade aplicada de potássio. Porém, 
como com NA houve redução do incremento de produção de MS com as doses de N (PRIMAVESI et al., 2003), a maior extração de $\mathrm{K}$ nesses tratamentos, com teores na forragem acima do adequado, pode significar início de consumo de luxo. Com os dois adubos e em todos os tratamentos, as extrações dos macronutrientes foram maiores para $\mathrm{K}$ seguidas de $\mathrm{N}, \mathrm{Ca}, \mathrm{Mg}, \mathrm{P}$ e $\mathrm{S}$ e dos micronutrientes na seguinte ordem decrescente: $\mathrm{Fe}, \mathrm{Mn}, \mathrm{Zn}$ e $\mathrm{Cu}$.

A recuperação aparente do $\mathrm{N}$ dos adubos variou $(\mathrm{P}<0,05)$ com as fontes e doses de $\mathrm{N}$ (Tabela 3). Com o aumento das doses de $\mathrm{N}$ ocorreu decréscimo na recuperação, maior com o NA. A recuperação média de todas as doses de N-uréia foi de $84 \%$ da obtida com o NA. $\mathrm{O} \mathrm{N}$-uréia recuperado pela parte aérea foi superior ao obtido por Oliveira et al. (2003). Esse fato foi devido provavelmente: a) à altura de corte de $10 \mathrm{~cm}$, que no ensaio de Oliveira et al. (2003) foi de $20 \mathrm{~cm}$; b) à dose de $\mathrm{N}$ usada de $400 \mathrm{~kg} \mathrm{ha}^{-1}$ em contraste com $300 \mathrm{~kg} \mathrm{ha}^{-1}$, utilizada por esses autores; c) e também ao uso pelos autores citados de ${ }^{15} \mathrm{~N}$, que elimina o efeito da $\mathrm{MO}$ (as raízes das plantas que receberam $\mathrm{N}$ exploram mais o $\mathrm{N}$ da $\mathrm{MO}$ do que as do tratamento sem N). Andrade et al. (1996), usando $1.150 \mathrm{~kg} \mathrm{ha}^{-1}$ de N como NA, relataram recuperação de $52 \%$, superior à deste trabalho, onde houve redução nas doses de $\mathrm{N}$ superiores a $400 \mathrm{~kg} \mathrm{ha}^{-1}$. Verificou-se uma baixa recuperação aparente do $\mathrm{N}$ nos terceiro e quarto períodos de crescimento (Tabela 3), podendo ser atribuída à baixa produção de forragem ocorrida, no terceiro período provavelmente pelas plantas já estarem florescidas e, no quarto, pela falta de chuvas.

TABELA 2 - Produção de matéria seca (MS) e extração mineral pelo capim-marandu em função de fontes e doses de $\mathrm{N}^{(1)}$.

\begin{tabular}{|c|c|c|c|c|c|c|c|c|c|c|c|}
\hline \multirow[t]{2}{*}{ Doses de $\mathbf{N}$} & \multirow{2}{*}{$\begin{array}{c}\text { MS } \\
\text { total }\end{array}$} & \multicolumn{10}{|c|}{ Nutrientes } \\
\hline & & $\mathbf{N}$ & $\mathbf{P}$ & $\mathbf{S}$ & $\mathbf{K}$ & $\mathbf{C a}$ & Mg & $\mathbf{C u}$ & $\mathbf{Z n}$ & Mn & $\mathbf{F e}$ \\
\hline $\mathrm{kg} \mathrm{ha}^{-1} \mathrm{ano}^{-1}$ & \multicolumn{7}{|c|}{ - } & \multicolumn{4}{|c|}{ - $\mathrm{g} \mathrm{ha}^{-1}$} \\
\hline & \multicolumn{11}{|c|}{ Uréia } \\
\hline 0 & 1.889 & 27 & 6 & 3 & 41 & 9 & 8 & 13 & 56 & 132 & 421 \\
\hline 200 & 6.650 & 112 & 23 & 8 & 196 & 30 & 25 & 41 & 212 & 365 & 1.100 \\
\hline 400 & 9.539 & 181 & 29 & 13 & 306 & 42 & 34 & 65 & 295 & 584 & 1.615 \\
\hline 800 & 12.328 & 282 & 36 & 17 & 429 & 48 & 44 & 84 & 424 & 863 & 2.032 \\
\hline média & 7.601 & 150 & 23 & 10 & 243 & 33 & 28 & 51 & 247 & 486 & 1.292 \\
\hline doses & $\mathrm{Q}^{* *}$ & $\mathrm{~L}^{* *}$ & $\mathrm{Q}^{* *}$ & $\mathrm{~L}^{* *}$ & $\mathrm{~L}^{* *}$ & $\mathrm{Q}^{* *}$ & $\mathrm{Q}^{*}$ & $\mathrm{~L}^{* *}$ & $\mathrm{~L}^{* *}$ & $\mathrm{~L}^{* *}$ & $\mathrm{~L}^{* *}$ \\
\hline \multirow[t]{2}{*}{$\mathrm{r}^{2} / \mathrm{R}^{2}$} & 0,99 & 0,99 & 0,99 & 0,99 & 0,99 & 0,99 & 0,99 & 0,99 & 0,99 & 0,99 & 0,99 \\
\hline & \multicolumn{11}{|c|}{ Nitrato de amônio } \\
\hline 0 & 1.889 & 27 & 6 & 3 & 41 & 9 & 8 & 13 & 56 & 132 & 421 \\
\hline 200 & 6.471 & 114 & 19 & 9 & 187 & 26 & 25 & 45 & 212 & 394 & 1.095 \\
\hline 400 & 11.481 & 229 & 36 & 17 & 380 & 44 & 43 & 88 & 386 & 666 & 2.075 \\
\hline 800 & 13.070 & 327 & 42 & 19 & 429 & 48 & 49 & 103 & 475 & 849 & 2.394 \\
\hline média & 8.228 & 174 & 26 & 12 & 259 & 32 & 31 & 62 & 282 & 510 & 1.496 \\
\hline doses & $\mathrm{Q}^{* *}$ & $\mathrm{~L}^{* *}$ & $Q^{*}$ & $\mathrm{Q}^{* *}$ & $\mathrm{Q}^{* *}$ & $\mathrm{Q}^{* *}$ & $\mathrm{Q}^{* *}$ & $\mathrm{Q}^{* *}$ & $\mathrm{Q}^{*}$ & $\mathrm{~L}^{* *}$ & $\mathrm{~L}^{* *}$ \\
\hline$r^{2} / R^{2}$ & 0,99 & 0,99 & 0,99 & 0,99 & 0,98 & 0,99 & 0,99 & 0,99 & 0,99 & 0,99 & 0,97 \\
\hline adubos & $* *$ & $* *$ & $*$ & $* *$ & $\mathrm{~ns}$ & ns & $* *$ & $* *$ & $* *$ & ns & $* *$ \\
\hline ad. $\mathrm{x}$ doses & $* *$ & $* *$ & $* *$ & $* *$ & $* *$ & $* *$ & $* *$ & $* *$ & $* *$ & $* *$ & $* *$ \\
\hline
\end{tabular}

${ }^{(1)}=$ média de quatro cortes. ${ }^{\text {ns }}=$ não-significativo.* ou ** = significância do teste $\mathrm{F}$ no nível de $5 \%$ e $1 \%$. Para doses, apresentada a curva de melhor ajuste $\left(\mathrm{L}=\right.$ Linear simples ou $\mathrm{Q}=$ Quadrática) e o valor de $\mathrm{r}^{2} / \mathrm{R}^{2}$. 
TABELA 3 - Extração e recuperação aparente de N pela parte aérea do capim-marandu em quatro períodos de cortes consecutivos.

\begin{tabular}{|c|c|c|c|c|c|c|c|c|c|c|}
\hline \multirow{3}{*}{$\begin{array}{c}\text { Doses de } \mathrm{N} \\
\text { kg ha }^{-1} \text { corte }^{-1}\end{array}$} & \multicolumn{5}{|c|}{ - } & \multicolumn{4}{|c|}{------- Recuperação de N ------- } & \multirow{4}{*}{ média } \\
\hline & $\mathbf{1}^{\mathbf{0}}$ & $2^{-}$ & $3^{\mathbf{o}}$ & $4^{0}$ & média & $\mathbf{1}^{\mathbf{0}}$ & $2^{\mathbf{o}}$ & $3^{\mathbf{o}}$ & $4^{0}$ & \\
\hline & \multirow{2}{*}{\multicolumn{9}{|c|}{ 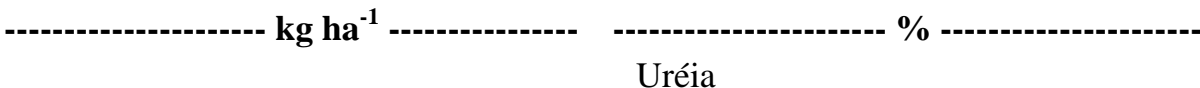 }} & \\
\hline & & & & & & & & & & \\
\hline 0 & 6 & 7 & 10 & 4 & 7 & - & - & - & - & - \\
\hline 50 & 30 & 42 & 27 & 14 & 28 & 48 & 69 & 34 & 19 & 43 \\
\hline 100 & 53 & 71 & 31 & 26 & 45 & 47 & 64 & 22 & 22 & 39 \\
\hline 200 & 108 & 94 & 51 & 29 & 71 & 51 & 43 & 21 & 13 & 32 \\
\hline Média & 49 & 53 & 30 & 18 & 38 & 49 & 59 & 16 & 18 & 37 \\
\hline Doses & - & - & - & - & $\mathrm{L}^{* *}$ & - & - & - & - & $\mathrm{L}^{*}$ \\
\hline \multirow[t]{2}{*}{$\mathrm{r}^{2} / \mathrm{R}^{2}$} & - & - & - & - & 0,99 & - & - & - & - & 0,98 \\
\hline & \multicolumn{10}{|c|}{ Nitrato de amônio } \\
\hline 0 & 6 & 7 & 10 & 4 & 7 & - & - & - & - & - \\
\hline 50 & 29 & 46 & 30 & 10 & 29 & 46 & 77 & 40 & 12 & 44 \\
\hline 100 & 62 & 91 & 49 & 27 & 57 & 56 & 83 & 40 & 23 & 51 \\
\hline 200 & 133 & 123 & 46 & 25 & 82 & 64 & 58 & 18 & 11 & 38 \\
\hline Média & 57 & 67 & 34 & 17 & 44 & 55 & 73 & 33 & 15 & 44 \\
\hline Doses & - & - & - & - & $\mathrm{L}^{* *}$ & - & - & - & - & $\mathrm{Q}^{* *}$ \\
\hline$r^{2} / R^{2}$ & - & - & - & - & 0,99 & - & - & - & - & 0,99 \\
\hline \multicolumn{11}{|l|}{ Teste F } \\
\hline Fontes de N & & & & & $* *$ & & & & & $*$ \\
\hline Interação & & & & & $*$ & & & & & ns \\
\hline
\end{tabular}

* ou ** = significância do teste $\mathrm{F}$ no nível de 5\% e 1\%. Para média das doses, apresentada a curva de melhor ajuste ( $\mathrm{L}=$ Linear simples ou $\mathrm{Q}=$ Quadrática) e o valor de $\mathrm{r}^{2} / \mathrm{R}^{2}$.

\section{CONCLUSÕES}

A extração de $\mathrm{K}$ é grande em capim-marandu com produções elevadas de forragem.

A recuperação média de $\mathrm{N}$ fornecido pela uréia é inferior a de $\mathrm{N}$ do nitrato de amônio, quando se utiliza pastagem de capim-marandu.

\section{REFERÊNCIAS BIBLIOGRÁFICAS}

ANDRADE, J. B.; BENINTENDE, R. B.; FERRARI JÚNIOR, E.; PAULINO, V. T.; HENRIQUE, V.; WERNER, J. C.; MATTOS, H. B. Nitrogênio e potássio na produção e composição de Brachiaria brizantha cv. Marandu. In: REUNIÃO BRASILEIRA DE ZOOTECNIA, 33., 1996, Fortaleza. Anais... Fortaleza: UFC, 1996. v. 2, p. 283-285.
ANDREW, C. S.; ROBINS, M. F. The effect of phosphorus on the growth, chemical composition, and critical phosphorus percentages of some tropical pasture grasses. Australian Journal of Agricultural Research, East Melbourne, v. 22, n. 5, p. 693-706, 1971.

BATISTA, K. Respostas do capim-marandu a doses de nitrogênio e enxofre. 2002. 91 f. Dissertação (Mestrado em Agronomia) - Escola Superior de Agricultura "Luiz de Queiroz”, Universidade de São Paulo, Piracicaba, 2002.

COUTINHO, E. L. M.; SILVA, A. R.; MONTEIRO, F. A.; RODRIGUES, L. R. A. Adubação potássica em forrageiras. In: SIMPÓSIO SOBRE MANEJO DA PASTAGEM, 21., 2004, Piracicaba. Anais... Piracicaba: FEALQ, 2004. p. 219-277. 
ENGELS, C.; MARSCHNER, H. Plant uptake and utilization of nitrogen. In: BACON, P. E. (Ed.). Nitrogen fertilization in the environment. New York: M. Dekker, 1995. p. 41-81.

FAQUIN, V.; LIMA, D. V.; FURTINI NETO, A. E.; MORAES, A. R.; CURI, N.; HIGA, N. T. Nutrição mineral do braquiarão e da soja cultivados em latossolos sob cerrado da região de Cuiabá, MT. Ciência e Agrotecnologia, Lavras, v. 24, p. 110-117, 2000.

GATES, C. E. A user's guide to misanalyzing planned experiments. HortScience, Alexandria, v. 26, n. 10, p. 12621265, 1991.

HOPKINS, A.; ADAMSON, A. H.; BOWLING, P. J. Response of permanent and reseeded grassland to fertilizer nitrogen: 2. effects on concentrations of $\mathrm{Ca}, \mathrm{Mg}, \mathrm{K}, \mathrm{Na}, \mathrm{S}$, $\mathrm{P}, \mathrm{Mn}, \mathrm{Zn}, \mathrm{Cu}, \mathrm{Co}$ and $\mathrm{Mo}$ in herbage at a range of sites. Grass and Forage Science, Oxford, v. 49, p. 9-20, 1994.

JARVIS, S. C.; SCHOLEFIELD, D.; PAIN, B. Nitrogen cycling in grazing systems. In: BACON, P. E. (Ed.). Nitrogen fertilization in the environment. New York: M. Dekker, 1995. p. 381-420.

JONES, W. F.; WATSON, V. H. Response of hybrid bermudagrass to sulfur application. Communications in Soil Science and Plant Analysis, New York, v. 22, p. 505-515, 1991.

LUZ, P. H. C.; HERLING, V. R.; PETERNELLI, M.; BRAGA, G. J. Calagem e adubação no manejo intensivo do pastejo. In: SIMPÓSIO DE FORRAGICULTURA E PASTAGENS: TEMAS EM EVIDÊNCIA, 2., 2001, Lavras. Anais... Lavras: UFLA, 2001. p. 27-110.

MALAVOLTA, E. Elementos de nutrição mineral de plantas. São Paulo: Ceres, 1980. 251 p.

MALAVOLTA, E.; VITTI, G. C.; OLIVEIRA, S. A. Avaliação do estado nutricional das plantas: princípios e aplicações. 2. ed. Piracicaba: POTAFOS, 1997. 319 p.

MARSCHNER, H. Mineral nutrition of higher plants. 2. ed. London: Academic, 1995. 889 p.

MATTOS, W. T. Diagnose nutricional de potássio nas Braquiárias decumbens e brizantha. 1997. 75 f. Dissertação (Mestrado em Agronomia) - Escola Superior de Agricultura “Luiz de Queiroz", Piracicaba, 1997.

MATTOS, W. T.; SANTOS, A. R.; ALMEIDA, A. A. S.; CARREIRO, B. D. C.; MONTEIRO, F. A. Aspectos produtivos e diagnose nutricional do capim-Tanzânia submetido a doses de potássio. Magistra, Cruz das Almas, v. 14, p. 37-44, 2002.

MONTEIRO, F. A.; COLOZZA, M. T.; WERNER, J. C. Enxofre e micronutrientes em pastagens. In: SIMPÓSIO SOBRE MANEJO DA PASTAGEM, 21., 2004, Piracicaba. Anais... Piracicaba: FEALQ, 2004. p. 279-301.

MONTEIRO, F. A.; RAMOS, A. K. B.; CARVALHO, D. D.; ABREU, J. B. R.; DAIUB, J. A. S.; SILVA, J. E. P.; NATALE, W. Cultivo de Brachiaria brizantha cv. Marandu em solução nutritiva com omissões de macronutrientes. Scientia Agricola, Piracicaba, v. 52, n. 1, p. 135-141, 1995.

NATIONAL RESEARCH COUNCIL. Nutrient requeriments of dairy cattle. 7 . ed. Washington: National Academy, 2001. 381 p.

OLIVEIRA, P. P. A.; TRIVELIN, P. C. O.; OLIVEIRA, W. S. Eficiência da fertilização nitrogenada com uréia $\left({ }^{15} \mathrm{~N}\right) \mathrm{em}$ Brachiaria brizantha cv. Marandu associada ao parcelamento de superfosfato simples e cloreto de potássio. Revista Brasileira de Ciência do Solo, Viçosa, v. 27, p. 613-620, 2003.

PRIMAVESI, A. C.; PRIMAVESI, O.; CORRÊA, L. A.; CANTARELLA,H.; SILVA,A. G; FREITAS,A. R.; VIVALDI, L. J. Adubação nitrogenada em capim-coastcross: efeitos na extração de nutrientes e recuperação aparente do nitrogênio. Revista Brasileira de Zootecnia, Viçosa, v. 33, n. 1, p. 68-78, 2004.

PRIMAVESI, O.; CORRÊA, L. A.; PRIMAVESI, A. C.; CANTARELLA,H.; SILVA, A. G. Adubação com uréia em pastagem de Brachiaria brizantha sob manejo rotacionado: eficiência e perdas. São Carlos: Embrapa-Pecuária Sudeste, 2003. 6 p. (Comunicado técnico, 41).

SCOTT, N. M.; WATSON, M. E.; CALDWELL, K. S. Response of grassland to the application of sulphur at two sites in north-east Scotland. Journal of the Science of Food and Agriculture, Edinburgh, v. 34, p. 357-361, 1983.

WERNER, J. C.; PAULINO, V. T.; CANTARELLA, H. Forrageiras. In: RAIJ, B. van; CANTARELLA, H.; QUAGGIO, J. A.; FURLANI, A. M. C. (Eds.). Recomendações de adubação e calagem para o Estado de São Paulo. Campinas: Instituto Agronômico, 1996. p. 263-273 (Boletim técnico, 100).

WHITEHEAD, D. C. Volatilization of ammonia. In:

Grassland nitrogen. Wallingford: CAB International, 1995. p. 152-179. 